\title{
Towards a comprehensive framework for cell-free DNA analysis: lessons from TRACERx
}

\author{
Bryan C. Ulrich ${ }^{1 *}$, Nicolas Guibert ${ }^{2}$ \\ ${ }^{1}$ Belfer Center for Applied Cancer Science, Dana-Farber Cancer Institute, Boston, MA, USA; ${ }^{2}$ Pulmonology Department, Larrey University \\ Hospital, France \\ *This author is currently at Emory School of Medicine, 1648 Pierce Dr. NE Atlanta, GA 30307, USA \\ Correspondence to: Dr. Nicolas Guibert, MD. Pulmonology Department, Larrey University Hospital, 24, chemin de Pouvourville, 31059 Toulouse \\ Cedex 9, Toulouse, France. Email: guibert.n@chu-toulouse.fr. \\ Provenance: This is a Guest Editorial commissioned by Section Editor Xue-Feng Leng (Department of Cardiothoracic Surgery, the Affiliated \\ Hospital of Chengdu University, Chengdu, China). \\ Comment on: Abbosh C, Birkbak NJ, Wilson GA, et al. Phylogenetic ctDNA Analysis Depicts Early-Stage Lung Cancer Evolution. Nature \\ 2017;545:446-51.
}

Submitted Jul 31, 2017. Accepted for publication Aug 04, 2017.

doi: 10.21037/atm.2017.08.12

View this article at: http://dx.doi.org/10.21037/atm.2017.08.12

\section{Introduction}

Molecular mechanisms of oncogenesis in lung cancer have been largely deciphered over the past 20 years. Cell-free DNA (cfDNA) is an emerging but immature clinical tool used to guide genotype-directed cancer care. Currently, FDA-approved clinical use of cfDNA is limited to the cobas EGFR Mutation Test v2 CE-IVD for non-small cell lung cancer (NSCLC) patients unable to undergo a tissue biopsy or with acquired resistance to EGFR-TKIs (1). However, many researchers and clinicians seek to expand the use of cfDNA to a wider range of clinical situations. Plasma genotyping assays are being explored in early detection, identifying minimal residual disease (MRD), monitoring treatment response, tracking resistance, and understanding tumor heterogeneity (2-7). Swanton et al., within the TRACERx [Tracking Non-Small-Cell Lung Cancer (NSCLC) Evolution Through Therapy (Rx)] Consortium, have initiated an extensive trial to assess cfDNA as a biomarker in each of these situations (8). Recently published preliminary TRACERx results provide deep insight into the biology of cfDNA shed and its potential clinical utility (9). Furthermore, the TRACERx team made significant advances in assay development which encourage a reformulation of variant calling in next-generating sequencing (NGS) assays that may improve the sensitivity and specificity of plasma genotyping assays.

\section{Key findings of the study}

The TRACERx team analyzed cfDNA data from the first 100 patients enrolled in the study from the point of diagnosis through treatment (including curative surgery) and beyond. Importantly, personalized sequencing assays were built and used for each patient. Multiregion exome sequencing of the tumor provided information on clonal and sub-clonal tumor variants. Using this information, personalized, patient-specific multiplex PCR assays were built for deep sequencing of these variants within the plasma. Although potentially costly and time-consuming, these assays had remarkable sensitivity (as many tumor variants were assayed by deep sequencing) and specificity (as the team set a threshold of 2 or more variants for determining the presence of tumor). Before further expounding on assay development, we will discuss the key findings of the preliminary results regarding early detection/ shed and MRD/recurrence post-curative surgery.

\section{Predictors of early detection of ctDNA}

Interest in the use of cfDNA to detect early-stage cancers has attracted funding and resources to several biotech companies, some of which have initiated large-scale trials to attempt to detect early cancer in high-risk populations using plasma genotyping (NCT02612350 and NCT03085888) (10). 
Detection of early-stage cancer via plasma genotyping requires tumor DNA shed and sufficiently sensitive assays to capture and characterize this circulating tumor DNA (ctDNA) amongst a sea of wild type DNA. Bettegowda et al. performed an extensive study on early-stage malignancies and determined that $47 \%$ of stage one cancers have detectable plasma ctDNA (11). Additionally, several studies have determined certain predictors of ctDNA shed, which include tumor size, number of metastatic sites, stage, and organ of origin $(3,4,12)$. This study aimed to further inform the incomplete understanding of ctDNA shed at diagnosis. Among the findings, the TRACERx team discovered necrosis, lymphovascular invasion, Ki67 labelling index, tumor size, and non-adenocarcinoma status all predict $\mathrm{ctDNA}$ shed. With their assay, they identified tumor cfDNA in $48 \%$ of early-stage NSCLC patients. Finally of note, the team discusses a theoretical limit of DNA shed for tumors of a given size, estimating that tumors under $10 \mathrm{~cm}^{3}$ will not have detectable circulating tumor DNA. This may limit the clinical utility of plasma genotyping in early detection. Overall, the TRACERx findings for early detection significantly improve our understanding of ctDNA shed biology and plasma genotyping as a diagnostic tool.

\section{Potential application to detect MRD and assess sub-clonal origin of recurrence}

In addition to ctDNA shed, important advances were made relating to ctDNA as a biomarker of $\mathrm{MRD} /$ recurrence in post-curative surgery patients. The utility of plasma genotyping in this context has been studied extensively in various malignancies $(13,14)$. These studies indicate that plasma genotyping of tumor variants in the plasma has high positive and negative predictive values and a lead time of weeks to months over the detection of MRD/recurrence via clinical assessment or imaging. This study builds on this work, showing that their assay has $93 \%$ sensitivity and $90 \%$ specificity for identifying recurrence with a lead time of 70 days over clinical/imaging follow-up. Interestingly, in many patients this assay identified the phylogenetic subclone that was responsible for recurrence. These data should motivate large-scale interventional studies that use cfDNA to inform clinical decision-making in this context.

\section{Comments}

Plasma genotyping assays have traditionally had difficulty at ascertaining the presence of tumor plasma cfDNA at low levels $[<0.5 \%$ allele frequency (AF)]. At these low levels, discordance between plasma and tumor genotyping, as well as discordance between orthogonal plasma genotyping assays, has been seen (15-18). This is particularly disadvantageous in the context of early detection and MRD/ recurrence, as false positives must particularly be avoided, and low AFs are typically seen in these contexts. Thus, assay performance at/near the limit of detection is important. The assay used in TRACERx is uniquely capable of reliability at very low AFs, with a sensitivity of above $99 \%$ and a specificity of $99.6 \%$ (for single variant calls) down to $0.1 \% \mathrm{AF}$. To our knowledge, this is the first study that sets a threshold of at least two tumor variants to determine that tumor plasma cfDNA is present. Other plasma genotyping assays often assess just the driver mutation, if known. With the TRACERx assay, it is possible that a highly confident call (e.g., a KRAS mutation at $2 \% \mathrm{AF}$ ) would be disregarded if in the absence of any other tumor variant assessed by the assay. Therefore, although individual variant calls are made independently of each other, dependent variant calling is necessary for the binary cancer/no cancer call. This is a fundamental advancement in variant calling in plasma NGS assays, and it supports further investigation into other dependent variant calling algorithms.

The TRACERx assay design and performance motivates variant calling that uses known clinical situations and patient characteristics to set AF thresholds for calls. Typically, we use highly confident NGS variant calls (> 0.5-1\% $\mathrm{AF})$ to inform clinical decision-making. For example, the finding of EGFR T790M (a common resistance mechanism to EGFR-TKI) in the plasma of a NSCLC patient on erlotinib would drastically increase the likelihood that such a patient would progress in a given time frame. However, the TRACERx study suggests the use of clinical knowledge to inform variant calling of lowly confident calls $(<0.5 \%$ $\mathrm{AF})$. For example, the finding of EGFR T790M at $0.1 \% \mathrm{AF}$ in a patient with EGFR-mutant NSCLC on erlotinib for some time has a higher likelihood of being a true positive than the same call in a patient with newly diagnosed KRASmutant NSCLC. Traditionally, variant calling thresholds are made by looking at a large population of admixtures/ patient plasma that you know do not have the mutation, and then setting $\mathrm{AF}$ thresholds so as to maximize sensitivity and specificity (often favoring specificity). However, this is imperfect as the presence of a mutation within the plasma is unknown in clinical situations, but the likelihood of certain mutations being present depends on the situation and patient characteristics. Therefore, it is possible to use 
clinical information to aid in variant calling. Formalizing such an idea is challenging, as it requires longitudinal monitoring of a specific large cohort of patients from diagnosis through treatment and beyond. All variant calls, no matter how low of AF, would have to be recorded and later correlated with outcome, thereby generating a dataset that can be used to generate AF thresholds for every clinical situation/patient characteristic.

In conclusion, the preliminary results of the plasma genotyping component of TRACERx make crucial insights into plasma genotyping assay development, the biology of cfDNA shed, heterogeneity in NSCLC, and the clinical utility of cfDNA to assess early detection and MRD/ recurrence. These data suggest that cfDNA is a biomarker with great clinical utility in diverse situations. In addition, they promote further investigation into different manners of variant calling in NGS assays. Clinical situationspecific variant calling is an intriguing potential solution to continued issues with sensitivity and specificity at low AFs. In this sense, the TRACERx study and other such longitudinal studies are especially qualified to formulate a comprehensive framework for cfDNA analysis.

\section{Acknowledgements}

None.

\section{Footnote}

Conflicts of Interest: The authors have no conflicts of interest to declare.

\section{References}

1. Center for Drug Evaluation and Research. Approved drugs-cobas EGFR Mutation Test v2 [Internet]. U S Food and Drug Administration Home Page. Center for Drug Evaluation and Research; Available online: https://www. fda.gov/Drugs/InformationOnDrugs/ApprovedDrugs/ ucm504540.htm

2. Murtaza M, Dawson SJ, Tsui DW, et al. Non-Invasive Analysis of Acquired Resistance to Cancer Therapy by Sequencing of Plasma DNA. Nature 2013;497;108-12.

3. Yanagita M, Redig AJ, Paweletz CP, et al. A Prospective Evaluation of Circulating Tumor Cells and Cell-Free DNA in EGFR-Mutant Non-Small Cell Lung Cancer Patients Treated with Erlotinib on a Phase II Trial. Clin Cancer Res 2016;22:6010-20.
4. Oxnard GR, Paweletz CP, Kuang Y, et al. Noninvasive Detection of Response and Resistance in EGFR-Mutated Lung Cancer using Quantitative Next-Generation Genotyping of Cell-Free Plasma DNA. Clin Cancer Res 2014;20:1698-705.

5. Murtaza M, Dawson SJ, Pogrebniak K, et al. Multifocal Clonal Evolution Characterized Using Circulating Tumour DNA in a Case of Metastatic Breast Cancer. Nat Commun 2015:6;8760.

6. Sundaresan TK, Sequist LV, Heymach JV, et al. Detection of T790M, the Acquired Resistance EGFR Mutation, by Tumor Biopsy vs Noninvasive Blood-Based Analyses. Clin Cancer Res 2016;22:1103-10.

7. Forshew T, Murtaza M, Parkinson C, et al. Noninvasive Identification and Monitoring of Cancer Mutations by Targeted Deep Sequencing of Plasma DNA. Sci Transl Med 2012;4:136ra68.

8. Cancer Research UK. TRACERx [Internet]. UK: Cancer Research UK; 2017. Available online: http://www. cruklungcentre.org/Research/TRACERx

9. Abbosh C, Birkbak NJ, Wilson GA, et al. Phylogenetic ctDNA Analysis Depicts Early-Stage Lung Cancer Evolution. Nature 2017;545:446-51.

10. NewswirePR. Guardant Health Announces Project LUNAR, An Ambitious Multi-arm, Multi-site Umbrella Trial to Study Blood Test for Early Cancer Detection [Internet]. U.S.: PR Newswire, 2016.

11. Bettegowda C, Sausen M, Leary RJ, et al. Detection of Circulating Tumor DNA in Early- and Late-Stage Human Malignancies. Sci Transl Med 2014;6:224ra24.

12. Sacher AG, Paweletz C, Dahlberg SE, et al. Prospective Validation of Rapid Plasma Genotyping for the Detection of EGFR and KRAS Mutations in Advanced Lung Cancer. JAMA Oncol 2016;2:1014-22.

13. Garcia-Murillas I, Schiavon G, Weigelt B, et al. Mutation Tracking in Circulating Tumor DNA Predicts Relapse in Early Breast Cancer. Sci Transl Med 2015;7:302ra133.

14. Tie J, Wang Y, Tomasetti C, et al. Circulating Tumor DNA Analysis Detects Minimal Residual Disease and Predicts Recurrence in Patients with Stage II Colon Cancer. Sci Transl Med 2016;8:346ra92.

15. Kuderer NM, Burton KA, Blau S, et al. Comparison of 2 Commercially Available Next-Generation Sequencing Platforms in Oncology. JAMA Oncol 2017;3:996-8.

16. Stetson D, Dougherty B, Ahmed A, et al. Examination of Analytical Factors Impacting Concordance of PlasmaTesting by Next Generation Sequencing (NGS). Presented at the Annual Conference of American Association for 
Cancer Research (AACR); April 2017; Washington D.C. 17. Schwaederlé MC, Patel SP, Husain H, et al. Utility of Genomic Assessment of Blood-Derived Circulating Tumor DNA (ctDNA) in Patients with Advanced Lung Adenocarcinoma. Clin Cancer Res 2017;23:5101-11.

18. Ulrich BC, Nagy RJ, Odegaard JI, et al. Cross-platform detection and quantification of actionable mutations in cell-free DNA shows high concordance and correlation between next-generation sequencing and droplet digital PCR [abstract]. In: Proceedings of the American Association for Cancer Research Annual Meeting 2017; 2017 Apr 1-5; Washington, DC. Philadelphia (PA): AACR; Cancer Res 2017;77:abstr nr 5692. doi:10.1158/15387445.AM2017-5692.
Cite this article as: Ulrich BC, Guibert N. Towards a comprehensive framework for cell-free DNA analysis: lessons from TRACERx. Ann Transl Med 2017;5(21):428. doi: 10.21037/atm.2017.08.12 